\title{
Chapter 2 \\ Updates in Treatment Modalities \\ and Techniques on Compromised Alveolar \\ Ridge Augmentation for Successful Dental Implant Therapy
}

\author{
Myung-Jin Kim
}

\begin{abstract}
Installation of dental implants is often hindered by compromised alveolar ridge which requires augmentation. Several methods including autogenous bone graft, guided bone regeneration, and distraction osteogenesis are implied to restore the compromised alveolar ridge. This chapter reviews treatment modalities and techniques of alveolar ridge augmentation. Recent development and updates in this field are also presented.
\end{abstract}

Keywords Alveolar ridge augmentation - Autogenous block bone grafting $\bullet$ Bone graft material $\bullet$ Distraction osteogenesis

\subsection{Treatment Modalities for Augmentation of the Compromised Alveolar Ridge}

Edentulism often exists with concomitant alveolar ridge resorption. Consequently implant installation is very often complicated with severely atrophic alveolar ridge. Various efforts have been tried to overcome the problem of insufficient alveolar bone for ideal implant installation. Alveolar bone augmentation which restores the atrophic alveolar bone to its near intact state will exhibit ideal properties in both functional and esthetic means for implant installation.

Alveolar bone resorption occurs in either a horizontal or vertical direction. A composite defect is also common. Treatment options for the horizontal alveolar bone defect include guided bone regeneration (GBR), veneer bone graft, ridge splitting technique, and distraction osteogenesis. For the vertical defect, the options are GBR, onlay bone graft, interpositional bone graft, and distraction osteogenesis.

\footnotetext{
M.-J. Kim (ه)

Department of Oral and Maxillofacial Surgery, Seoul National University Dental Hospital, 101, Daehak-ro, Jongno-gu, Seoul 110-768, Republic of Korea

e-mail: myungkim@snu.ac.kr 


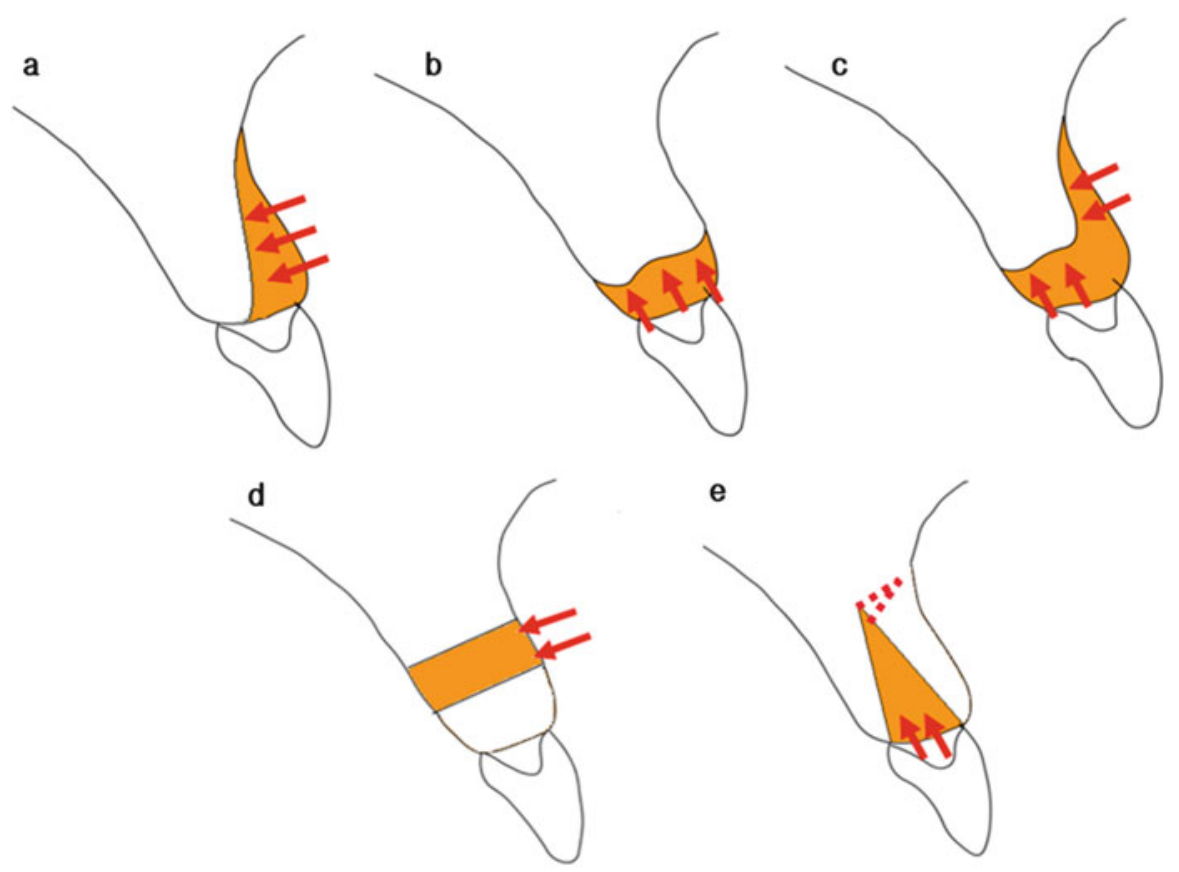

Fig. 2.1 Schematic diagrams of alveolar ridge augmentation. (a) Veneer bone graft, (b) onlay bone graft, (c) saddle bone graft, (d) interpositional bone graft, (e) ridge splitting technique

The composite defects can be restored by GBR, saddle bone graft, or distraction osteogenesis (Fig. 2.1).

Bone restoration capability differs according to the method applied. Guided bone regeneration is restricted in terms of small feasible bone regain amount. When applied with resorbable membranes, the expected bone regeneration does not exceed 3-4 mm vertically and $4 \mathrm{~mm}$ horizontally. Titanium mesh may enhance the capability to 4-7 $\mathrm{mm}$ vertically and $5 \mathrm{~mm}$ horizontally. Onlay block bone graft has similar capability with the latter of GBR technique. Interpositional bone graft is capable of regaining 5-7 mm of vertical height. Distraction osteogenesis is the most capable modality in terms of feasible bone regain amount which may exceed $10 \mathrm{~mm}$ in vertical height and $6 \mathrm{~mm}$ in alveolar width.

\subsection{Autogenous Block Bone Grafting}

Autogenous bone graft had been the gold standard of bone grafting. It is the only graft material that is considered to have osteoconductive, osteoinductive, and osteogenetic properties all together. Furthermore when block bone is adapted properly, it may contribute to initial stability of the implant fixture installed 


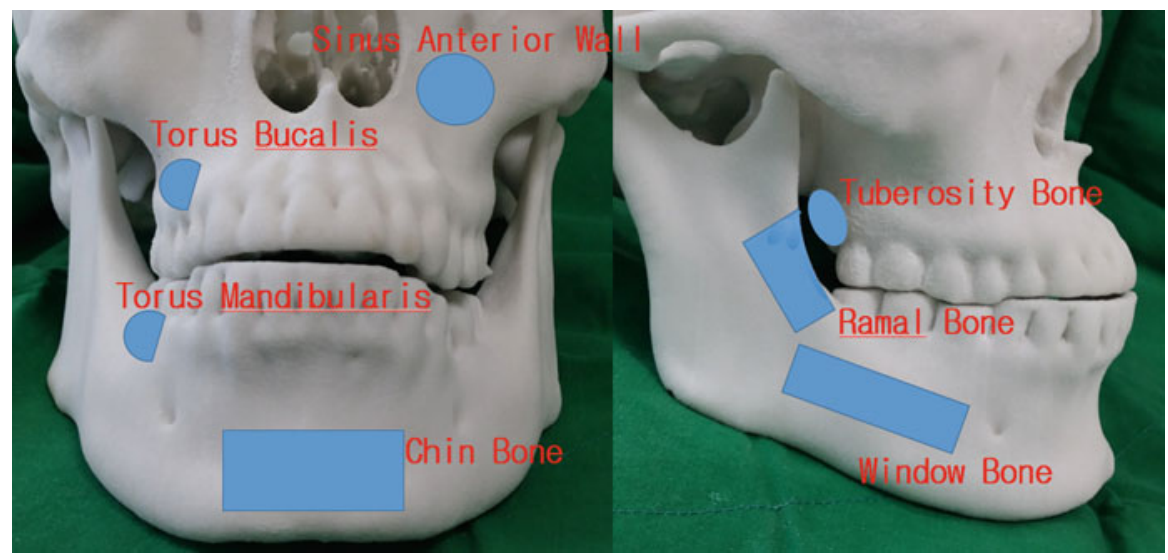

Fig. 2.2 Schematic diagram of the intraoral donor sites of autogenous bone

simultaneously. This characteristic can increase the feasibility of simultaneous bone grafting and implant installation.

\subsubsection{Autogenous Bone Donor Sites}

Autogenous bone is harvested from either an intraoral site or extraoral. Intraoral donor sites include the chin bone, the ramal bone, the mandibular body window bone, the mandibular torus bone, the maxillary torus bone, the sinus anterior wall and the maxillary tuberosity bone. The chin bone is the most abundant source among the listed (Figs. 2.2 and 2.3).

Extraoral donor sites include the anterior iliac crest bone, the proximal tibia bone, and the calvarial bone. These sites may suffice any amount of requisite for an alveolar augmentation.

\subsubsection{Block Bone Grafting Techniques}

The most intuitive form of autogenous block bone grafting is the onlay or veneer grafting techniques (Fig. 2.4). These techniques directly replace the resorbed alveolar bone in a vertical or horizontal direction, respectively. Both vertical and horizontal augmentations are possible by the saddle bone graft technique. Khoury demonstrated the method and results of sinus floor augmentation with mandibular block bones where dental implants were installed simultaneously [1]. Cases with severely atrophic alveolar ridge may require additional bone grafting. In the atrophic maxilla, subnasal or subantral block bone graftings are applied in addition of the onlay bone graft for this purpose. 

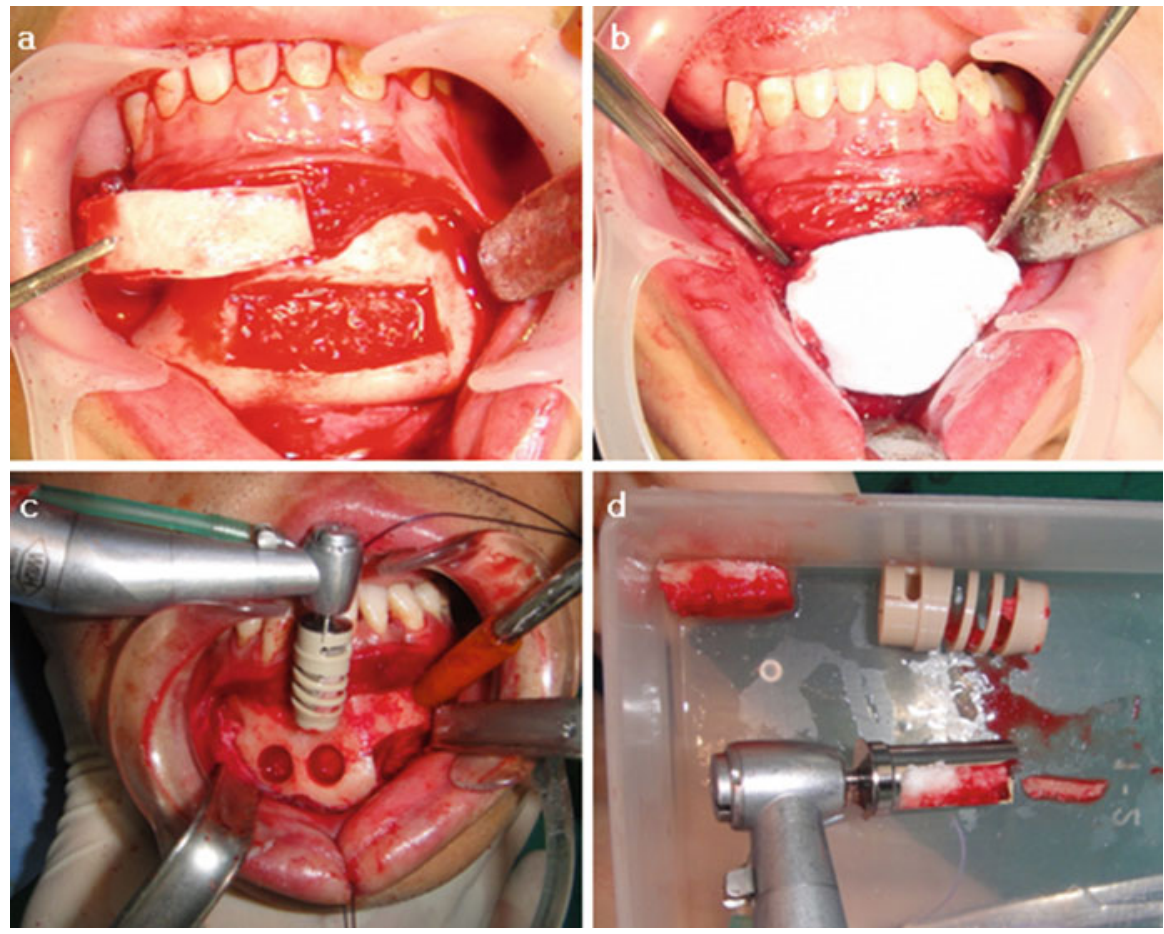

Fig. 2.3 Chin bone harvesting. (a) Window type block bone harvesting, (b) Barrier membrane placed for bone healing of the donor site, (c and d) Mushed particulated bone block harvesting with bone mill bur $\left(\right.$ Neobiotech $\left.{ }^{\circledR}\right)$

Vertical interpositional bone graft is an alternative to the conventional methods described above, when the bone demand is large. This method has the advantage of preserving the alveolar crest with intact nourished bone. Soft tissue management and flap design is very important and should be done carefully with interpositional bone graft procedures for preserving the vascularity.

\subsubsection{The Fate of Autogenous Onlay Bone Graft}

Resorption of grafted autogenous bone is always a matter of concern. Cordaro et al. reported that when block bones harvested from the mandibular ramus or symphysis are grafted in an onlay style, mean resorption rates during a graft healing period of 6 months were $23.5 \%$ for lateral grafts and $42 \%$ for verticals [2]. Proussaefs et al. suggested that vertical augmentation of the alveolar ridge with autogenous block bone from the mandibular ramus is a viable treatment option. They reported a vertical resorption rate of $16.34 \%$ from an initial $6.12 \mathrm{~mm}$ of augmentation after 4-6 months of healing period [3]. Widmark 

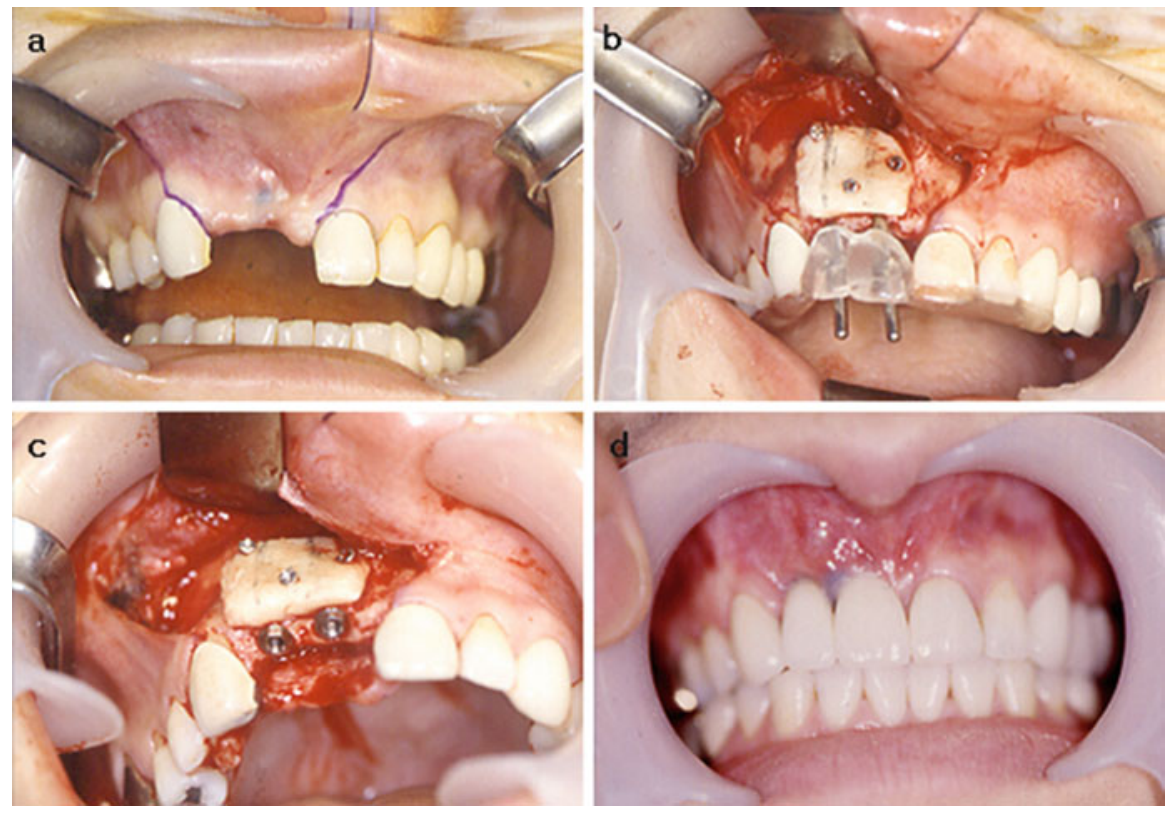

Fig. 2.4 Case presentation of a veneer bone graft. (a) Edentulous state on the anterior maxilla with atrophic and narrow alveolar ridge and the flap design, (b) Veneer bone graft positioned and fixed with titanium screws, (c) Implant installation, (d) Final prosthetic outcome

et al. demonstrated that the horizontal resorption was $25 \%$ after 4 months and $60 \%$ at the time of abutment connection when symphyseal block bone was grafted to the anterior maxilla for single tooth implant installation [4].

In a clinical survey conducted in our department, 76 patients underwent simultaneous autogenous onlay bone graft and installation of total 256 dental implants from 2000 to 2006. The amount of bone augmentation was $5.42 \mathrm{~mm}$ in average. After a minimum follow up period of 2 years, average bone resorption at the mesial and distal site of implant collar was $1.48 \mathrm{~mm}$. About $27.3 \%$ of the augmented bone height was resorbed.

\subsubsection{Drawbacks of Autogenous Bone Grafting}

Although the autogenous bone is considered as the gold standard of bone grafting for its osteogenic potential, drawbacks exist which limits its application. Donor site morbidity is of the most concern. Bone harvesting procedures may put adjacent anatomical structures at a risk of damage. For instance during chin bone harvesting, the mental nerve may be pulled under undue traction and the incisive nerve may become interrupted when the harvesting depth is inordinate. Ramal bone harvesting can damage the inferior alveolar nerve. 
In addition some patients may be reluctant to the harvesting procedures, especially when extraoral donor sites are concerned. General anesthesia is mandatory for these procedures. Gate problem could occur when autogenous bone is harvested from the tibia or the iliac crest.

\subsection{Biomaterials for Guided Bone Regeneration}

Guided bone regeneration utilizes the principle concept of guided tissue regeneration which is to separate a space from ingrowth of unintended tissues. Addition of bone graft materials in the space can secure the space from collapsing and promote the speed of bone regeneration. Bioengineering techniques may either enhance osteoinductive properties or mimic osteogenesis in non-autogenous graft materials (Fig. 2.5).

\subsubsection{Bone Graft Materials}

Bone graft materials can be classified according to their source of origin. Each class possesses different capability for regeneration of bone. The most ideal graft material in terms of regeneration potency is the autogenous bone graft which has osteogenetic properties. However application of autogenous bone graft may be restricted. Furthermore, in particular instances of graft material under constant

Fig. 2.5 A schematic diagram of the guided bone regeneration technique, $(a)$ Mucoperiosteal flap, $(b)$ GBR membrane, $(c)$ Bone graft material, (d) Host bone bed

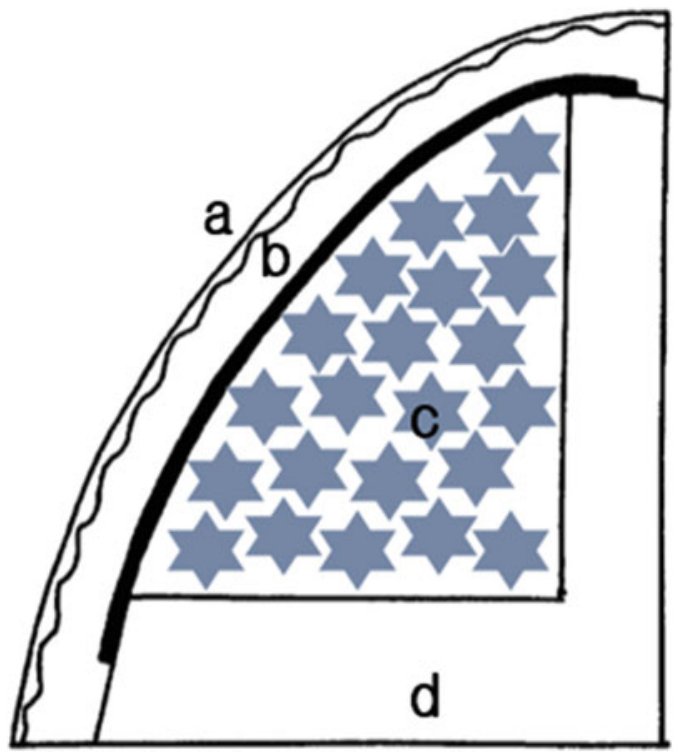


pressure, the autogenous bone graft may fail to secure the graft space. The rapid turnover rate may be problematic. In summary, non-autogenous bone grafts are not simply alternatives to autogenous but may be the best option we have for specific cases.

In Korea, various non-autogenous graft materials are commercially available for clinical use. These allogenic, xenogenic and alloplastic bone graft materials are recommended for small minor bone defects in combination with the guided bone regeneration technique.

\subsubsection{Allogenic Bone Graft}

Allogenic bone graft material originates from human individuals other than the recipient individual, genetically. Bone banks typically accumulate allogenic bone grafts from two donor sources, namely cadaveric and living donors who are undergoing bone removal procedures. Three forms of allogenic bone graft are available. Fresh or fresh-frozen allograft, freeze dried bone allograft and demineralized freeze dried bone allograft. When applying allografts for bone augmentation, the risk of disease transmission is of concern. Fortunately, according to the Centers for Disease Control and Prevention, there have been no reports of disease transmission when freeze-dried types of allograft are used for periodontal procedures.

Advantage of the allogenic grafts over the other bone graft materials is that these grafts can carry osteoinductive properties. Xenogenic and alloplastic bone graft materials are only osteoconductive at the best, unless they are enhanced by bioengineering techniques.

Allogenic bone graft materials available and currently in clinical use in Korea are as in the following. Demineralized freeze-dried form of allografts are Grafton ${ }^{\circledR}$, Orthoblast II ${ }^{\circledR}$, Tutoplast ${ }^{\circledR}$, Regenafil ${ }^{\circledR}$, and SureFuse ${ }^{\circledR}$. Freeze-dried bones include Puros $^{\circledR}$, OraGraft $^{\circledR}$ and $\mathrm{ICB}^{\circledR}$.

\subsubsection{Xenogenic Bone Graft}

Xenogenic bone grafts are harvested and processed from other species than human. Bovine bone is the most representative. This class of bone graft materials is accepted to have only osteoconductive properties. Commercially available products include Bio-Oss ${ }^{\circledR}$, BioCera ${ }^{\circledR}$, Biogen ${ }^{\circledR}$, Osteoplant ${ }^{\circledR}$, OSC$^{-} \mathrm{B}^{\circledR}$, and NuOss ${ }^{\circledR}$.

Both allogenic and xenogenic bone graft materials can be applied for subantral augmentation. Regardless of the residual alveolar bone height, if it provides sufficient initial stability for the implant fixture, bone graft and implant installation can be performed simultaneously. If initial stability is not obtained, implant installation should be delayed for a recommended period of 9 to 12 months after subantral bone graft. 


\subsubsection{Alloplastic Bone Graft}

Alloplastic bone graft materials are usually synthetic in its origin and are known to have only osteoconductive properties. Products consisting of sole or combinations of hydroxyapatite, calcium carbonate and beta tricalcium phosphate are available. Examples from Korean markets are Calcitite $\mathrm{HA}^{\circledR}$, Osteon, Osteograft ${ }^{\circledR}$, Frios ${ }^{\circledR}$, Algipore $^{\circledR}$, OsteoGen ${ }^{\circledR}$, HA Resorb ${ }^{\circledR}$ for sole hydroxyapatite, CeraSorb ${ }^{\circledR}$, InduCera $^{\circledR}$, Biobase ${ }^{\circledR}$ for sole $\beta$-TCP, MBCP ${ }^{\circledR}$ for combination of hydroxyapatite and $\beta$-TCP, and Biocoral ${ }^{\circledR}$, Interpore ${ }^{\circledR}$, SIC nature bone ${ }^{\circledR}$ for sole calcium carbonate.

Alloplastic bone graft materials are often produced in combination with osteoinductive substances in Korea and sold in the market. Hydroxyapatite mixed with rhBMP-2 such as Novosis ${ }^{\circledR}, \beta$-TCP mixed with rhBMP-2 such as Cowell $\mathrm{BMP}^{\circledR}$, and products containing biphasic calcium phosphate lyophilized with rhBMP-2 materials are also available. $\beta$-TCP mixed with rhBMP-2 and bio-degradable Hydrogel is produced as an injectable putty with a trade name Exelos Inject ${ }^{\circledR}$.

\subsubsection{Bioengineering Techniques}

Ideal bone graft materials should exhibit not only osteoconductive properties but as well osteoinductive. They should also provide a favorable environment for the invading blood vessels and bone forming cells. Recently various basic and clinical researches on the effect of recombinant human bone morphogenetic protein 2 (rhBMP-2) and potential use of human multipotent mesenchymal stromal/stem cells (MSC) are being reported and investigated.

\subsubsection{1 rhBMP-2}

Bone morphogenetic protein was first introduced by Urist in 1965 as a consisting substance of a decalcified bone [5]. Several different types of the protein are classified now with approval of products containing bone morphogenetic protein 2 and 7 for orthopedic application by the FDA.

Current focus of investigation concerning rhBMP-2 is concentrated on the carrier of the growth factor. Carriers of rhBMP-2 are required to have properties that will make possible to control the release of rhBMP-2 to the bone defect. Uncontrolled release of rhBMP-2 not only diminishes its effectiveness but also may induce concentration related side effects. Currently in our institute, both combination of collagen hydrogel, nano-hydroxyapatite and rhBMP-2 coated on dental implant surface and poloxamer based hydrogel with rhBMP-2 are under investigation. 


\subsubsection{Autologous Bone Marrow Mesenchymal Stem Cells}

Bone graft materials can be enhanced by addition of stem cells which may improve the rate and quality of defect repair. MSC can be isolated from the bone marrow or the periosteum and may be cultured for expansion. They can differentiate into several types of cell lines including fibroblast, chondroblast, endothelial cell, and of our most concern osteoblast which may accelerate bone regeneration. Derivation of mesenchymal stem cells from the bone marrow is the best characterized approach for osteogenic differentiation. The iliac spine is an easily manipulated, abundant store of autologous bone marrow MSC. Their use in combination with bone graft materials as a scaffold has produced promising clinical results (Fig. 2.6).

\subsection{Alveolar Ridge Augmentation by Distraction Osteogenesis}

In general, the available amount of soft tissue for wound closure restricts the capacity of bone grafting. Soft tissue coverage is crucial. Otherwise infection over the graft material may occur. Graft materials could be lost. At the best, the attained bone augmentation will be far behind than required.

Alveolar distraction osteogenesis (DO) is the most recent approach for alveolar ridge augmentation with promising results. Unlike any other approaches for alveolar ridge augmentation, DO is capable of lengthening both the hard and soft tissue. This method has the advantage of preserving the transport part of alveolar crest with intact vascularized nourished bone. This property permits the amount of alveolar augmentation attained by DO to surpass what the conventional methods of bone grafting would possibly do.

\subsubsection{Distraction Techniques}

Alveolar DO is capable to regenerate bone in both vertical and horizontal direction. Vertical distraction is achieved by placing the osteotomy line in a horizontal direction. By placing the osteotomy line at a more basal position of the alveolar ridge, a greater amount of width of regenerated bone is achievable. Horizontal distraction of the alveolar ridge utilizes the ridge splitting technique for the osteotomy. Takahashi et al. developed a novel method of applying a titanium mesh plate on the split transport segment and a rod for widening the gap which was known 'Alveo Wider' [6] (Fig. 2.7).

The span of the edentulous alveolar ridge should be at least two teeth wide in purpose to apply this technique. Otherwise the transport segment is under a risk of resorption during distraction. When the span of the alveolar ridge that requires 

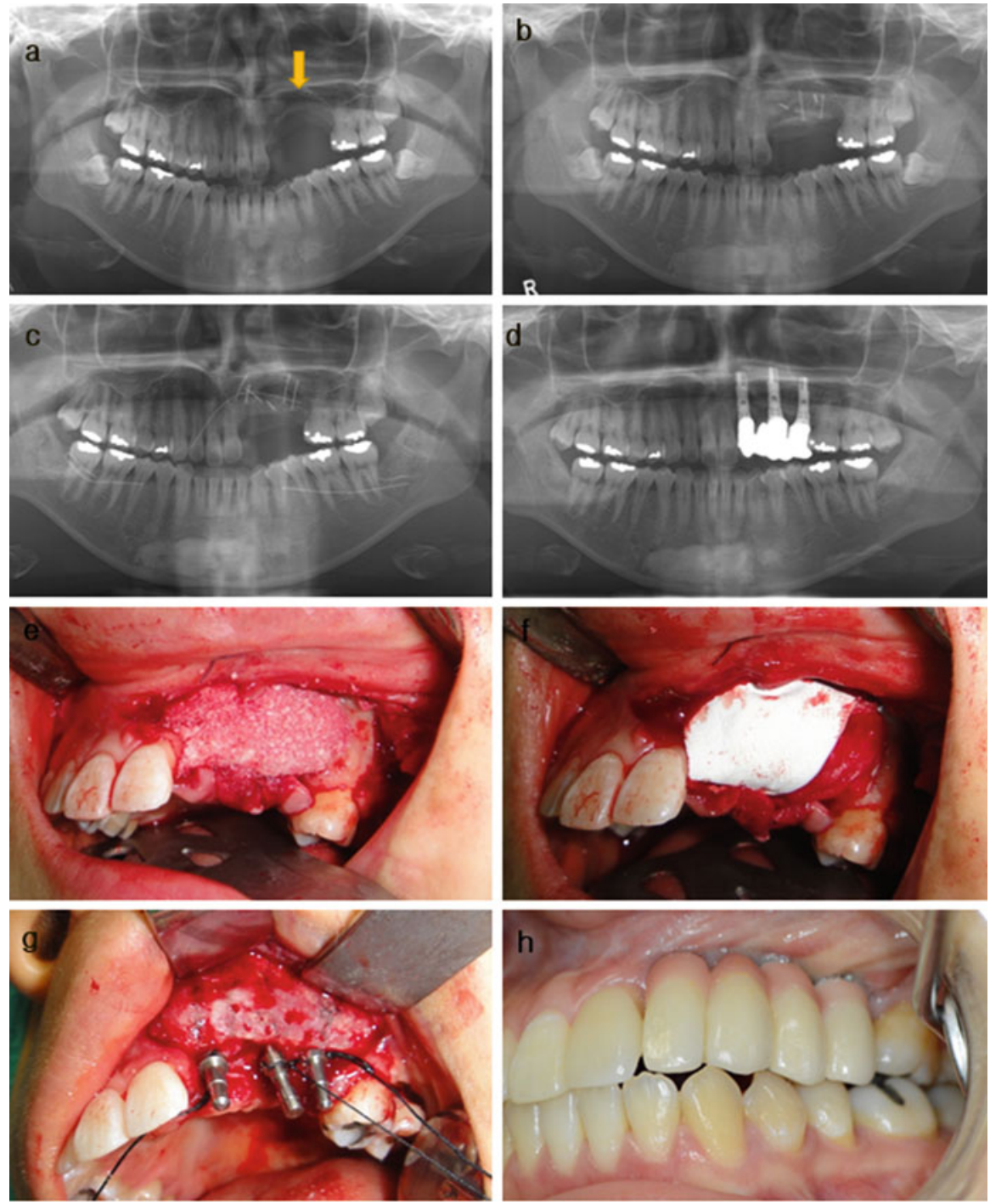

Fig. 2.6 A case presentation of an alveolar basal bone reconstruction. (a) Severe alveolar bone defect following resection of adenomatous odontogenic tumor on the left anterior and pre-maxilla depicted by an arrow, (b) First stage operation was done for reconstruction of basal part of the alveolar bone defect by onlay bone graft, veneer bone graft, and xenogenic bone graft mixed with chin bone, (c) X-ray finding shows Xenogenic bone graft mixed with autologous MSC at second stage operation, (d) Implant installation 6 months after 2nd stage bone grafting procedure, (e) Second stage operation was done for the reconstruction of alveolar part of the bone defect by a combination of ramal bone graft and guided bone regeneration with xenogenic bone graft mixed with autologous bone marrow MSC, (f) Barrier membrane placed over the graft, (g) Augmented alveolar bone at the stage of implant installation, (h) Final outcome 

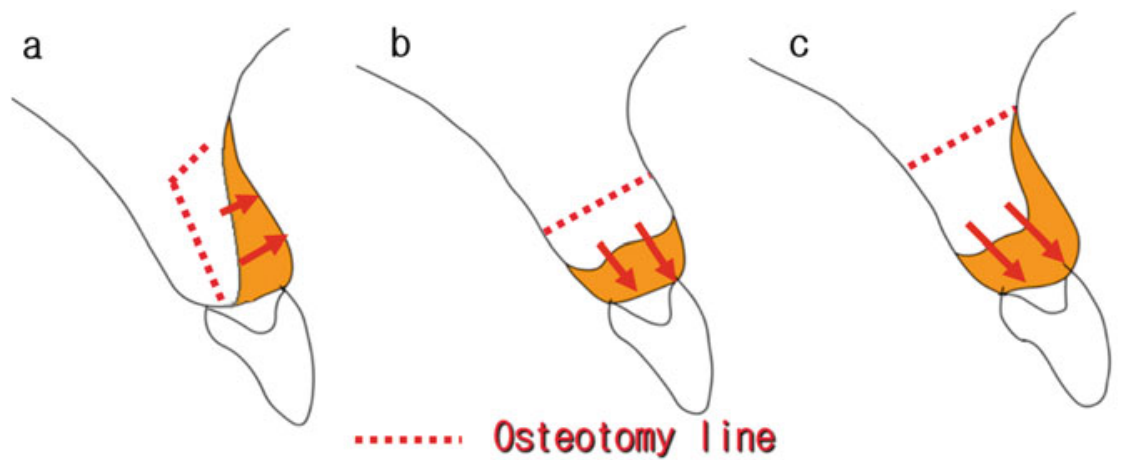

Fig. 2.7 Schematic diagrams of alveolar DO. (a) Horizontal DO, (b) Vertical DO, (c) Vertical DO for vertical and horizontal bone augmentation

distraction becomes larger, it can be segmented according to the vector of distraction required.

The alveolar distraction timeline requires that the latency period be $3-5$ days postoperatively. The distraction velocity should be adjusted according to the span of the transport segment. Short segments should be distracted slowly. This is intended to minimize the resorption of the transport segment. Two times of $0.3 \mathrm{~mm}$ distraction per day will satisfy this intention. With larger transport segments, three times of $0.3 \mathrm{~mm}$ distraction or two times of $0.5 \mathrm{~mm}$ distraction per day is allowed. After the distraction period, 3 months of consolidation period is required before implant installation (Figs. 2.8, and 2.9).

\subsubsection{Possible Amount of Bone Gain: Clinical Outcome}

In a clinical study reported by Paeng et al. in 2006, 25 patients who have undergone alveolar ridge augmentation by DO and subsequent installation of total 84 dental implants were investigated. Average amount of augmentation was $9.8 \pm 3.4 \mathrm{~mm}$. All of the implants installed survived for an average follow up period of 13.5 months. They suggested that DO may be acknowledged as a viable treatment option for alveolar augmentation so far [7]. In another case series of four patients who underwent alveolar distraction of the anterior atrophic mandible for dental implants, Yeom et al. reported that an average of $11.38 \pm 1.38 \mathrm{~mm}$ vertical gain was obtained by distraction [8]. DO can also be applied for augmentation of a reconstructed mandible with vascularized fibular free flap. Paeng et al. stressed that despite a tendency of surgical site infection during distraction of the fibular bone, undisturbed bone regeneration occurs for successful dental implant installation [9]. 

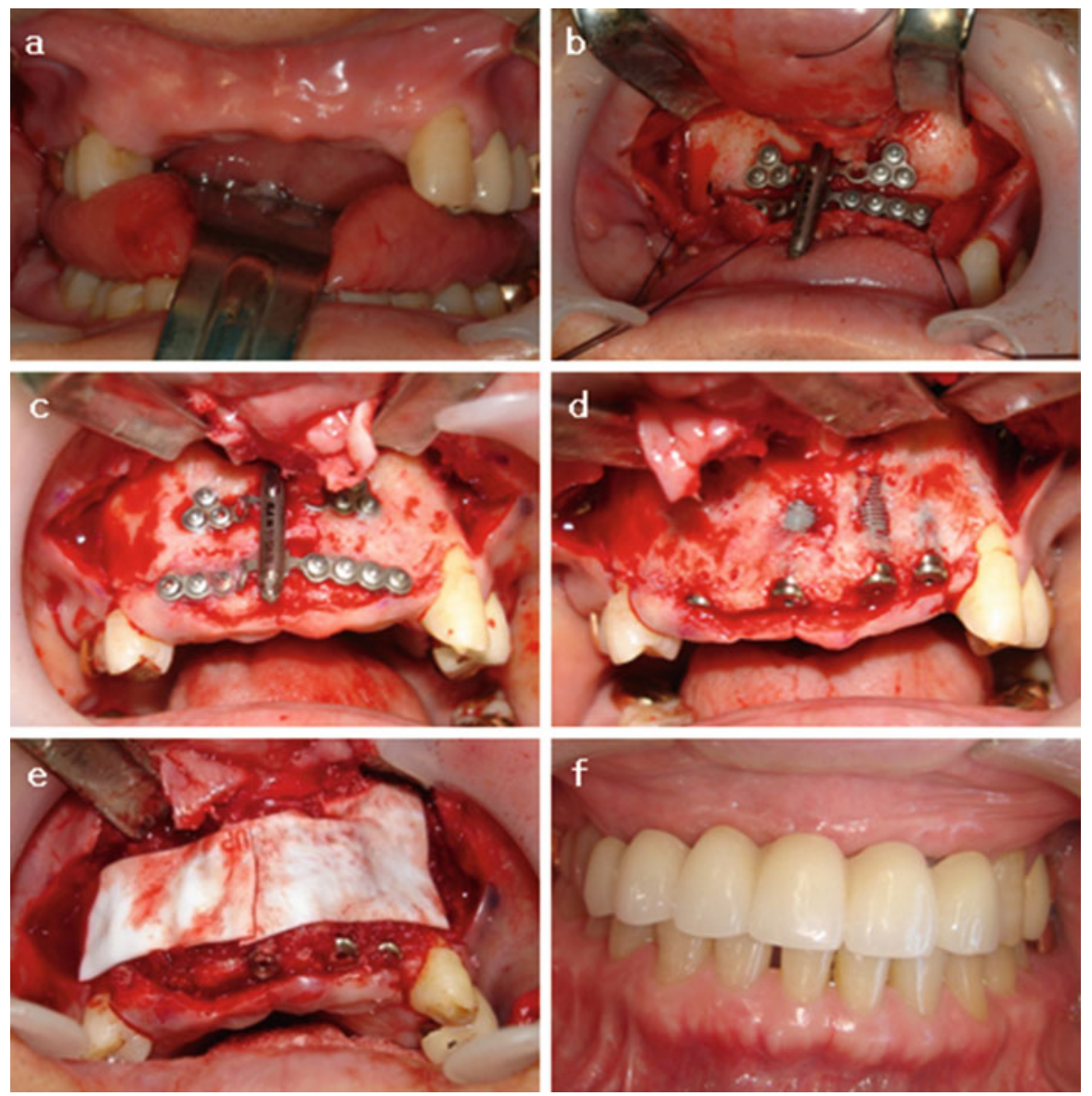

Fig. 2.8 Case presentation of an alveolar DO of the anterior maxilla. (a) Preoperative atrophic edentulous state of the anterior maxilla, (b) Installation of the distraction device, (c) Regenerated bone by distraction osteogenesis after 4 months postoperatively, (d) After implant installation, (e) Guided bone regeneration technique applied for the repair of bony fenestrations, (f) Final outcome

\subsubsection{Consolidation Enhancement Factors}

Current studies on DO of the alveolar bone are focused on the consolidation enhancement factors. The purpose of these studies is to develop a novel method to shorten the consolidation time and at the same time to enhance the mechanical property of the newly distracted bone. Both biological and mechanical stimulations are under research.

Kim et al. reported that significant amount of new bone volume was observed when human mesenchymal stromal cells were injected to the distraction site one day before distraction initiation in a rabbit mandibular distraction model. Human mesenchymal stromal cells also increased bone mineral density [10]. 

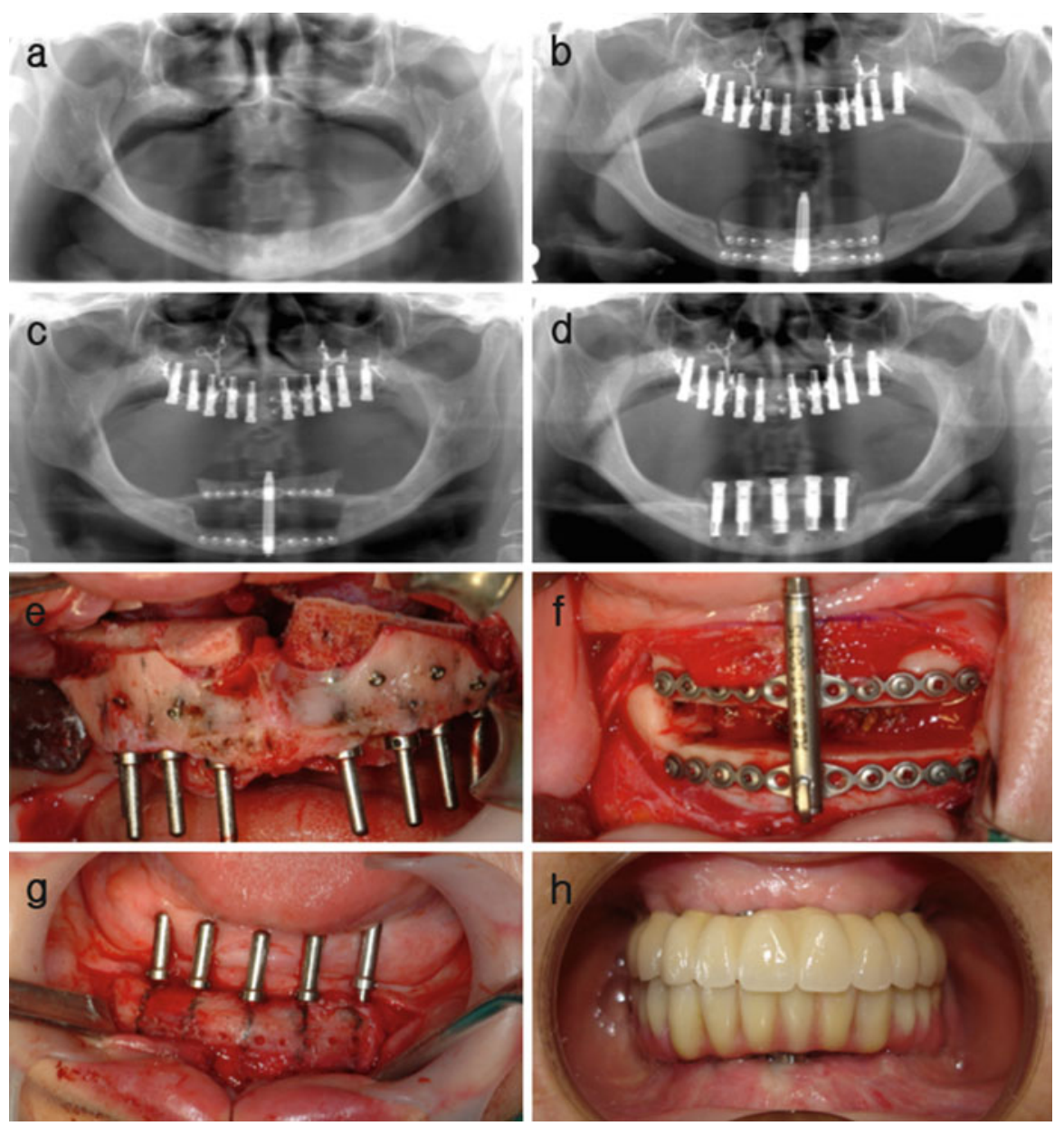

Fig. 2.9 Case presentation of a severe maxillo-mandibular atrophy. (a) Radiograph representing severe atrophy of both the maxilla and the mandible, (b) After Le Fort 1 osteotomy and iliac bone graft with simultaneous implant installation of the maxilla and distraction device installation on the mandible, (c) After full activation of the distraction device, (d) After implant installation on the mandible, (e) Clinical photographs of the operation. Maxilla was down fractured and moved anteriorly and interpositional iliac bone graft was performed and fixed rigidly on the preplanned position and implant installation was done simultaneously, (f) Activation trial of the distraction device at the time of installation, (g) Regenerated bone on the distracted alveolar bone on the mandible and implants were installed, (h) Final prosthetic outcome

Growth factors are also candidates. Floerkemeier et al. demonstrated that percutaneous triple injection of rhBMP-2 improves trabecular microarchitecture of the regenerated bone and in turn contributes to advanced mechanical integrity [11]. Fugio et al. suggested that failure of high speed DO attributes to lack of bone marrow endothelial cells and endothelial progenitor cells into the gap formed by 
distraction. In their study, stromal cell derived factor 1 showed possibility to overcome the resulting ischemic condition and facilitate acceleration of distraction speed [12].

Mechanical means of consolidation enhancement include electric, laser, and ultrasonic stimulations. Hwang et al. investigated the effect of pulsed electromagnetic field stimulation on consolidation of the distracted callus with results of enhanced bone formation. In their study, they applied the pulsed electromagnetic field stimulation for 5 days at the beginning of the consolidation period [13]. Miloro et al. studied the effect of low-level laser on DO. They found that lower-level laser when applied after each activations of distraction device accelerates bone regeneration [14]. The effect of low-intensity pulsed ultrasound when applied during the consolidation period had been reported by Shimazaki et al. They reported that not only normal distraction protocol but also a rapid distraction protocol may benefit from the application of low-intensity pulse ultrasound [15].

Acknowledgement The author would like to thank professor Jong-Ho Lee, Soon-Jung Hwang, Soung-Min Kim and doctor Kang-Mi Pang, Ju-Hyun Kim and Seung-Ki Min of the School of Dentistry, Seoul National University for their help provided in preparing this manuscript.

Open Access This chapter is distributed under the terms of the Creative Commons Attribution Noncommercial License, which permits any noncommercial use, distribution, and reproduction in any medium, provided the original author(s) and source are credited.

\section{References}

1. Khoury F. Augmentation of the sinus floor with mandibular bone block and simultaneous implantation: a 6-year clinical investigation. Int J Oral Maxillofac Implants. 1999;14(4):557.

2. Cordaro L, Amade DS, Cordaro M. Clinical results of alveolar ridge augmentation with mandibular block bone grafts in partially edentulous patients prior to implant placement. Clin Oral Implants Res. 2002;13(1):103-11.

3. Proussaefs P, Lozada J, Kleinman A, Rohrer MD. The use of ramus autogenous block grafts for vertical alveolar ridge augmentation and implant placement: a pilot study. Int J Oral Maxillofac Implants. 2002;17(2):238.

4. Widmark G, Andersson B, Ivanoff C-J. Mandibular bone graft in the anterior maxilla for single-tooth implants: presentation of a surgical method. Int J Oral Maxillofac Surg. 1997;26 (2):106-9.

5. Urist MR. Bone: formation by autoinduction. Science. 1965;150(3698):893-9.

6. Takahashi T, Funaki K, Shintani H, Haruoka T. Use of horizontal alveolar distraction osteogenesis for implant placement in a narrow alveolar ridge: a case report. Int J Oral Maxillofac Implants. 2004;19(2):291.

7. Paeng JY, Myoung H, Hwang SJ, Seo BM, Choi JY, Lee JH, et al. Clinical evaluation of alveolar distraction osteogenesis for implant installation. J Korean Assoc Maxillofac Plast Reconstr Surg. 2006;28(4):329-38.

8. Yeom HR, Jeon SH, Kim YT, Paeng JY, Ahn KM, Myung H, et al. Implant installation using vertical distraction osteogenesis at a severely atrophied edentulous mandible. J Korean Assoc Maxillofac Plast Reconstr Surg. 2006;28(2):154-65. 
9. Paeng JY, Lee JY, Myoung H, Hwang SJ, Seo BM, Choi JY, et al. Vertical distraction osteogenesis for implant installation on the reconstructed mandible with free fibular flap. J Korean Assoc Maxillofac Plast Reconstr Surg. 2006;28(6):579-85.

10. Kim IS, Cho TH, Lee ZH, Hwang SJ. Bone regeneration by transplantation of human mesenchymal stromal cells in a rabbit mandibular distraction osteogenesis model. Tissue Eng Part A. 2013;19(1-2):66-78. doi:10.1089/ten.TEA.2011.0696.

11. Pastor MF, Floerkemeier T, Witte F, Nellesen J, Thorey F, Windhagen H, et al. Repetitive recombinant human bone morphogenetic protein 2 injections improve the callus microarchitecture and mechanical stiffness in a sheep model of distraction osteogenesis. Orthop Rev. 2012;4(1):e13. doi:10.4081/or.2012.e13.

12. Fujio M, Yamamoto A, Ando Y, Shohara R, Kinoshita K, Kaneko T, et al. Stromal cell-derived factor-1 enhances distraction osteogenesis-mediated skeletal tissue regeneration through the recruitment of endothelial precursors. Bone. 2011;49(4):693-700. doi:10.1016/j.bone.2011. 06.024.

13. Hwang KK, Cho TH, Song YM, Kim DK, Han SH, Kim IS, et al. Effect of pulsed electromagnetic field stimulation on the early bone consolidation after distraction osteogenesis in rabbit mandible model. J Korean Assoc Maxillofac Plast Reconstr Surg. 2007;29(2):123-31.

14. Miloro M, Miller JJ, Stoner JA. Low-level laser effect on mandibular distraction osteogenesis. J Oral Maxillofac Surg. 2007;65(2):168-76. doi:10.1016/j.joms.2006.10.002.

15. Shimazaki A, Inui K, Azuma Y, Nishimura N, Yamano Y. Low-intensity pulsed ultrasound accelerates bone maturation in distraction osteogenesis in rabbits. J Bone Joint Surg. 2000;82(7):1077-82. 